\title{
A Taxonomy of Challenges for Cloud ERP Systems Implementation
}

\author{
Qian Huang* Misita Anwar Gillian Oliver Mahbubur Rahim \\ Monash University \\ Qian.Huang@monash.edu
}

\begin{abstract}
Though success stories of Cloud Enterprise Resource Planning systems (Cloud ERP) are frequently published, the failure rate of Cloud ERP is relatively less reported and discussed. Implementing Cloud ERP systems is not always easy, and at times very challenging. Hence, client organizations and vendors should be aware of challenges to help ensure successful implementation of Cloud ERP systems. The aim of this study is to develop a taxonomy of Cloud ERP implementation challenges. A set of thirty-one challenges was identified from a systematic literature review, and were then categorized by following the taxonomy development process proposed by Nickerson et al. (2013). The taxonomy consists of two dimensions: type of challenges and locus of challenges. Our proposed taxonomy has implications for providing a springboard for further theory development in the Cloud ERP domain
\end{abstract}

\section{Introduction}

Enterprise Resource Planning (ERP) systems represent a comprehensive software package that aims to integrate the holistic processes of business and functions for the purpose of presenting a comprehensive view of organizations from a single information and IT architecture [1]. In recent years, owing to the emergence of Cloud Computing technologies, ERP vendors have begun offering a new breed of ERP systems by capitalizing the enormous potential of those technologies. Cloud ERP refers to ERP applications integrated with Cloud infrastructure and delivered over the Internet to multiple client organizations [2]. Cloud ERP is quite easy for client organizations' to deploy and does not require extensive IT infrastructure in terms of software and hardware [3]. It allows companies with limited budgets a more affordable way to access the technology. As a result, Cloud ERP systems are gaining popularity for SMEs as well as large organizations [4]. Despite such benefits, the failure rate of Cloud ERP implementation is a major concern [5]. Hence, researchers from different disciplines are paying increased attention to various aspects of Cloud ERP implementation [6].

Cloud ERP implementation refers to the process of offering and using an efficient Cloud ERP through configuring technical, organizational, and financial resources [7]. According to [8], the existing literature on Cloud ERP implementation addresses three key topical areas: critical success factors (CSFs) (e.g.[9],[8]), challenges (e.g.[10]) and benefits (e.g.[3]). However, very few systematic literature analyses on Cloud ERP implementation challenges has been undertaken. The journey of organizations during Cloud ERP implementation is difficult because many challenges (e.g., relating to security and privacy) are encountered [11]. To successfully address the difficulties associated with Cloud ERP implementation and achieve the promised benefits, it is necessary to understand related challenges. This provides the motivation for this study.

While some researchers have endeavored to investigate challenges affecting Cloud ERP implementation, their findings are to some extent inconsistent. Existing literature identifies challenges that hamper Cloud ERP implementation. Those challenges however have not yet been rigorously categorized for improved understanding of Cloud ERP implementation phenomenon.

A taxonomy provides the means to organize knowledge and increase understanding in discussion, pedagogy and research [12]. A taxonomy is used not only to systematically describe the current development or relationships of a research area, but also as a foundation for higher-order theory development such as a theory for explaining and predicting in an examined field [13]. The main observation for the high degree of complexity in Cloud ERP implementation is the different stakeholders involved. Vendors are the first to be contacted by client organizations for any new requirements or if they encounter a problem. At the same time, vendors have to host the function and the quality of systems for many client organizations [3, 14]. Different roles may result in different types of challenges for each stakeholder [15]. A taxonomy is useful to explain challenges of Cloud ERP implementation since its multidimensionality lays the foundations for understanding and analyzing challenges 
in terms of the dimensions and characteristics [12]. However, no such taxonomy has emerged from the existing literature for the context of Cloud ERP implementation.

The aim of this study is to develop a taxonomy for challenges affecting Cloud ERP implementation success identified from the existing literature. Our taxonomy development process followed the iterative approach proposed by Nickerson and Varshney [15]see figure 1. Both the conceptual to empirical (C2E) and the empirical to conceptual (E2C) methods were used. This paper contributes to theory and practice. As a theoretical contribution, the proposed taxonomy delivers a set of systematic and structured challenges for internal and external organizations (i.e., client organizations, Cloud ERP vendors) alike. This will help managers to synthesize and systematize research on challenges of Cloud ERP implementation. Furthermore, our proposed taxonomy can be considered as a Type 1 theory for analysis that lays the foundation for further theory development in the domain of Cloud ERP [13]. As a contribution to practice, our proposed taxonomy can provide practitioners with taxonomy of challenges to contextually understand as well as consider the challenges while undertaking Cloud ERP implementation. Moreover, it helps client organizations and vendors to focus on addressing more important individual challenges that they may encounter during Cloud ERP implementation according to their role. For example, decision makers in client organizations can pay more attention to address the first three challenges in one sub-category of the taxonomy (internal strategic challenge) during the development of management strategies. They also can consider external challenges when they develop and measure SLA performance of vendors. For vendors, their priority might be to, overcome external challenges to optimize services for client organizations. Without such a taxonomy, it would be difficult and time consuming for anyone to consider implementation challenges comprehensively.

The paper is organized as follows: first, the methodology applied to carry out the systematic literature review is explained. Then the findings section is structured into three subsections based on issues related to challenges followed by discussion. The paper concludes with implications for practice and research.

\section{Research approach}

A two-stage methodology has been followed. Stage 1 involves conducting a systematic literature review (SLR) to identify a list of Cloud ERP challenges from the extant literature. Stage 2 involves following a taxonomy development process as proposed by Nickerson and Varshney [15].

\subsection{A systematic literature review (SLR)}

A SLR is a process of identifying, assessing and interpreting all available literature produced by researchers. The study reported in this paper, was conducted by drawing on the method of SLR introduced by Jones and Gatrell [16]. To identify and select appropriate papers for review, a predefined set of keywords and a set of inclusion/exclusion criteria was used to reduce selection bias [17]. A flow chart of SLR is shown in Figure 1.

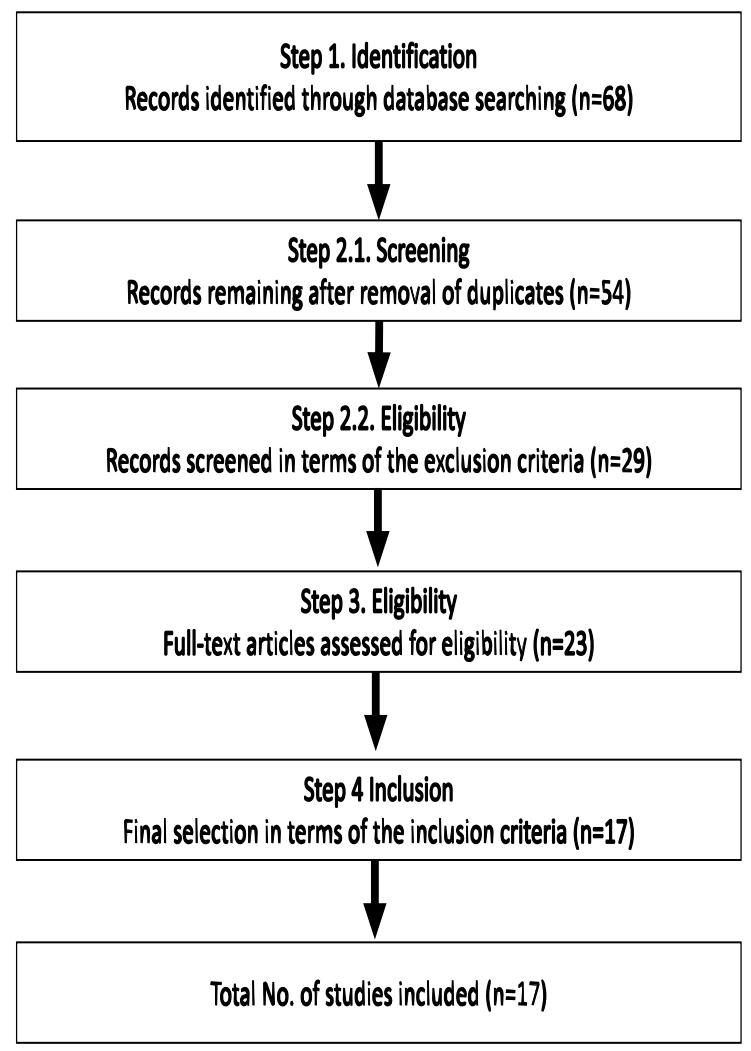

Figure 1. SLR flow

Our research began with identifying relevant papers from the AIS "basket of eight" journals (i.e. Information Systems Journal, Information Systems Research, MIS Quarterly, European Journal of Information Systems, Journal of the AIS, Journal of Information Technology, Journal of Management Information Systems, Journal of Strategic Information Systems) and leading IS conferences (i.e. PACIS, HICSS, ECIS, AMCIS, ICIS). To cover a broad a set of publications, and check the coverage, digital databases (i.e. Science Direct, JSTOR, ProQuest and Google scholar) were also searched.

Four steps were involved in the search and selection process. In step 1, keywords were identified and used 
for searching. The term "Cloud ERP" was used as a primary search term. In order to identify as many relevant papers as possible, alternative expressions of the primary search term were also used: "Cloud based ERP", "CERP”. A set of secondary keywords were also used for searching: "implementation" (and as an alternative the closely related term: "adoption") and "challenges" (and as alternatives the closely related words: "barriers", "impediments"). The Boolean operators (i.e. AND, OR and NOT) were applied to make the search accurate and more effective. In total, sixty-eight papers were identified from step 1. In step 2, fourteen papers were identified as duplicates and removed. Furthermore, twenty-five papers were excluded that did not meet the following four criteria: a) not clearly related to our research focus (i.e. the challenges for Cloud ERP implementation), b) not published in English, c) not published between 2010 and 2021, and d) not published as journal articles or conference papers. Other materials, such as blogs and workshop proposal, were excluded.

The main selection process focused on step 3 and step 4. In step 3, initial selection was conducted by reviewing title, abstract, and keyworks. The full texts of papers were manually evaluated by authors to ensure the chosen papers met the focus of this paper (i.e., challenges of Cloud ERP implementation). In step 4, the following selection criteria were adopted to select the candidate papers. First, challenges of Cloud ERP implementation are explicitly mentioned and are the focus of the papers' investigation. Second, since this paper only focuses on the implementation process, papers regarding the organizational decision making of Cloud ERP adoption were excluded. Third, challenges themselves are the primary focus or prominently discussed. A set of seventeen (17) papers was identified after step 4.

Out of seventeen, ten papers were published in journals. Five papers were published in 2018 whereas four papers were published in 2012. Twelve papers applied a literature review in their research design to investigate challenges in Cloud ERP implementation, but very few papers reported an empirical study.

\subsection{Following a taxonomy development process}

The taxonomy development approach proposed by Nickerson and Varshney [15] has been chosen for our research for three reasons. First, this method integrates empirical and conceptual perspectives into one comprehensive method that facilitates iteration for both perspectives. Second, objective and subjective criteria for the ending conditions have been clearly provided.
Third, this method is developed for IS and has been successfully applied by several IS researchers $[12,18]$.

According to Nickerson and Varshney [15], taxonomy development includes seven steps. Steps 1 and 2 determine the research field and the boundaries of the taxonomy. The purpose of steps 3 to 6 is to define and validate the taxonomy's characteristics and dimensions iteratively. A decision was made about whether another iteration should be conducted through comparing to the ending conditions in step 7. Our taxonomy process applied both the $\mathrm{C} 2 \mathrm{E}$ and the $\mathrm{E} 2 \mathrm{C}$ approach based on existing literature about challenges of Cloud ERP implementation. In the $\mathrm{C} 2 \mathrm{E}$ approach, the dimensions of the taxonomy were from the conceptual or theoretical foundation associated with Cloud ERP implementation challenges [19]. In the E2C approach, dimensions of the taxonomy were derived by analyzing specific challenges of Cloud ERP implementation and detecting their similarities or distinctions [19]. An overview of the applied research steps is shown in Figure 2.

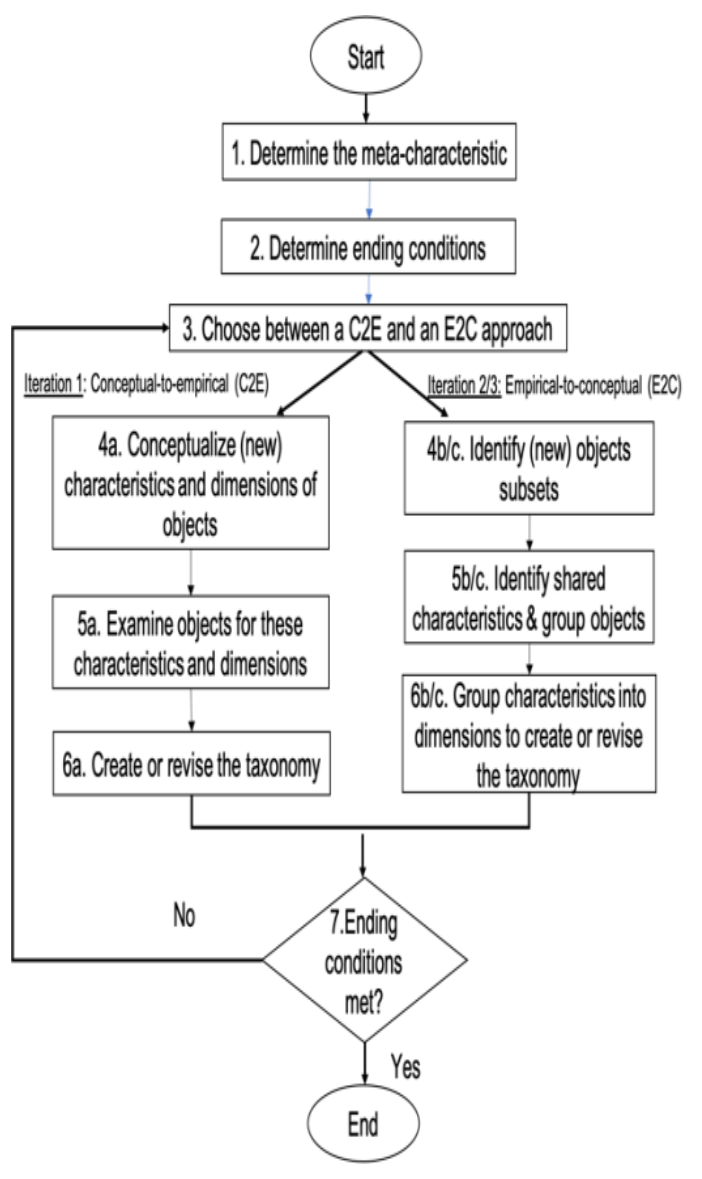

Figure 2. Applied Taxonomy Development Method of Nickerson, Varshney [20] 


\section{Findings}

The findings are expressed by identifying a set of thirty-one (31) challenges from Stage 1, which were then used for developing a taxonomy based on Stage 2 (as described earlier).

\subsection{List of Challenges}

We analyzed the selected seventeen (17) papers and identified a total of thirty-one (31) challenges associated with Cloud ERP implementation that have been discussed in existing literature. Out of these, the most important five (5) challenges were: data security, customization, network failure, service level agreement, long term cost. These frequently cited challenges are discussed below.

Data security and privacy is the most frequently reported challenge. Organizations are generally concerned about how organizational and customers' data can be protected as they are stored in a server which is located beyond organizational boundaries. In Cloud ERP, sensitive information about organizations (e.g., financial data, bank details) has to be shared with a third-party service where data can be mingled with that of other companies. Because of the openness and multitenant feature of the Cloud, client organizations are concerned about how secure their data is and who has access [2]. As a result, it is a major challenge for Cloud ERP vendors to offer strong protection from misuse and hacking [21].

Customization is the second most frequently reported challenge. As systems offered by Cloud ERP vendors are based on best business practices and do not take into consideration the unique process of each client organization, customization is required for client organizations to achieve the alignment between their business processes/requirements and a Cloud ERP [22]. However, any extensive customization is very costly [3]. This is especially considered as a deterrent for large organizations to implement systems because large organizations generally have more complex systems and processes compared to SMEs [4, 23].

The next most frequently reported challenge is Network and Internet Failure. Cloud ERP requires a constant internet connection for real-time transactions to take place. Network and Internet failure will have negative influence on organizations. For example, a slow or erratic internet connection may cause delays in transferring data, which may further result in slower decision making [4]. The handling of massive Internet traffic during a distributed denial of service (DDoS) attack is always a significant challenge for vendors. They are required to provide some feasible solutions to prevent the situation where client organizations are unable to access the Cloud server because of DDoS attack [2].

Another frequently reported challenge relates to Service Level Agreements (SLAs). SLAs are often proposed by Cloud ERP vendors, to include quality, availability and performance of the offered services [3]. However, SLAs are usually insufficient to cover all confidentiality issues or to attribute responsibility for damage liability [24]. More penalties and checking procedures for SLA are required from the customer perspective. More comprehensive SLAs are beneficial for both customers and providers and facilitate the implementation and maintenance of systems [25]. Therefore, it is challenging for client organizations to ensure the comprehensiveness of SLAs

Finally, long-term cost is found to be a frequently reported challenge for Cloud ERP implementation. Gupta and Misra [4] suggest that long-term cost is a major challenge for large organizations to implement Cloud ERP because the incremental amount of data requires on going purchasing of more storage space for systems. The cost is also regarded as a challenge for the context of SMEs in implementing systems since the annual subscription cost of Cloud ERP is higher than the maintenance costs of on-premise ERP. Large enterprises generally do not have budget issues compared to SMEs. Accordingly, annual subscription costs can be a challenge for SMEs to implement Cloud ERP systems [4, 26].

\subsection{A Taxonomy of Cloud ERP Challenges}

By carefully following a seven-step process [20], we now propose the development of a taxonomy of Cloud ERP challenges.

Step 1 (Determine the meta-characteristic): A meta-characteristic serves as the most comprehensive traits of entities and represents the main purpose of a taxonomy [27]. This step helps researchers and guides the development process to avoid examining some unrelated characteristics. For our Cloud ERP context, the meta-characteristic represents the characteristics of challenges for Cloud ERP implementation.

Step 2 (Determine the ending conditions): The development process will end in terms of objective and subjective ending conditions proposed by Nickerson and Varshney [19]. The objective ending conditions include the addition of no new dimensions in the last iteration and no additional challenges requiring examination. The development process will end when all of the subjective ending conditions are met, that is, when the taxonomy is determined to be comprehensive, concise, robust and explanatory, and extendible.

\section{Iteration 1:}


Steps 3 to 7: For the first iteration, we decided to use the $\mathrm{C} 2 \mathrm{E}$ approach since one dimension could be extracted from the existing literature of Cloud ERP implementation challenges (Step 3). Although there exists a little discussion on this topic for the Cloud ERP context, types of Cloud ERP challenges (in terms of citations) proposed by Saeed and Juell-Skielse [27] is the most frequently cited work. According to the authors, challenges are grouped into three different characteristics: a) strategic challenge is related to organizations' strategies, which is reflected by lack of early adopters because of low awareness of Cloud ERP, heavy investment in on-premise ERP, and heavily dependent on vendor; $b$ ) operational challenge is related to the operation of the system, which is reflected in terms of inconsistency between current business strategy and Cloud ERP, huge hidden costs, loss of jobs for in-house IT employees, and c) technical challenge is related to technical aspect of the system, which reflected security and privacy issue, the difficulty in customization and integration (Step 4a). In step 5a, some instances of these characteristics of challenges were identified from the existing literature. For example, strategic risk is a strategic challenge since client organizations may lose their own competitive advantages resulting from heavily depending on vendors and complying with their policies [28]. Longterm costs are an operational challenge because some hidden costs of Cloud ERP implementation may be higher than the running cost for on-premise ERP [3]. Integration is a technical challenge because Cloud ERP is difficult to integrate with the existing applications or IT infrastructure [29]. Accordingly, these three characteristics were added in our taxonomy in step 6a. Given that one dimension was created in this iteration, the development process had to be repeated to comply with the objective ending conditions (i.e., no new dimensions are added in the last iteration) (step 7).

\section{Iteration 2:}

Steps 3 to 7: the E2C approach was used in the second iteration because some challenges were identified from the Cloud ERP literature, which might provide additional dimensions for this taxonomy (step 3 ). We selected the first ten widely cited challenges from existing literature: security and privacy, customization, network and internet, SLA and longterm costs, performance, elasticity and scalability, functionality fit, integration and compliance (step 4b). One dimension, the locus of challenges (i.e. internal and external challenges) was identified based on our understanding of these challenges. Internal challenges refer to those challenges which arise among internal stakeholders (i.e. client organization) whereas external challenges refer to those challenges which are faced or likely faced by external stakeholders (i.e. Cloud ERP vendors) in dealing with Cloud ERP implementation). For example, Cloud ERP vendors are required to solve security issues because they have the ownership of Cloud ERP and client organizations cannot access and improve IT infrastructures by themselves [2]. Client organizations need to estimate the possible running costs and it is hard for them to identify some hidden costs for implementing Cloud ERP [4]. Accordingly, security is an external challenge whereas long-term costs is an internal challenge (step 5b). This dimension was added to the taxonomy in step 6b. However, the method had to be repeated because of two reasons: first, the locus of challenge was created in this iteration, which did not satisfy the objective ending conditions; second, it is not known if the taxonomy is comprehensive enough because more challenges exist that are required to be considered. As a result, one more iteration is needed (step 7).

\section{Iteration 3:}

Steps 3 to 7: As there are more challenges to examine, the $\mathrm{E} 2 \mathrm{C}$ approach was followed for this iteration (step 3). The remaining twenty-one challenges from the literature were considered, such as reliability of systems, organizational change (step 4c). We could not identify any new dimensions from these challenges. These challenges could be classified into the existing dimensions (steps $5 c, 6 c$ ). Hence, no new dimensions were added in this iteration, and all challenges extracted from existing literature had been examined. In conclusion, the objective ending conditions are met. Furthermore, the taxonomy appears to be comprehensive, concise, robust and explanatory after extracting some dimensions and examining the considerable number of challenges from existing literature. As a result, it meets the subjective ending conditions. The development process ends at this point.

In summary, our proposed taxonomy (Figure 3 ) is described in terms of two dimensions: type of challenges and locus of challenges. Drawing on this taxonomy, a set of six categories of challenges is identified. Our assumption underlying this taxonomy, is that organization size does not influence both these dimensions. For example, SMEs and large client organizations do not differ from each other on the selection of vendors. This is because vendors are responsible for looking after data of client organizations and then it can be difficult to change the vendor [4]. Furthermore, the performance of Cloud ERP and the network connectivity has been always the matter of concern with both SMEs and large organizations [4]. 


\begin{tabular}{|c|c|c|c|}
\hline \multicolumn{4}{|c|}{ Locus of Challenges } \\
\hline \multirow{3}{*}{ 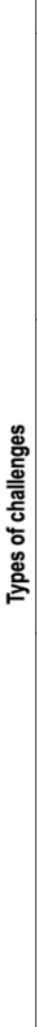 } & & \begin{tabular}{|l|}
\multicolumn{1}{|c|}{ Internal } \\
Category A: Internal-Strategic \\
challenge \\
- Strategic risk [3],[28] \\
- Integrity of provider [4] \\
- Management Awareness [4] \\
- Perception about cloud ERP \\
using [4],[28] \\
- Organizational culture and the \\
structure of the organization \\
[33] \\
\end{tabular} & \begin{tabular}{|l}
\multicolumn{1}{|c|}{ External } \\
Category B: External-Strategic challenge \\
- Compliance risks [22],[23],[25],[28] \\
- The strategy development for facilitating \\
Cloud ERP [28] \\
- Legal issue [4]
\end{tabular} \\
\hline & 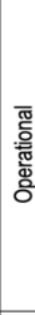 & $\begin{array}{l}\text { Category C: Internal-Operational } \\
\text { challenge } \\
\text { - SLAs [24],[23],[33],[3],[28],[40] } \\
\text { - Long-term costs } \\
\text { [4],[33],[3],[25],[28],[40] } \\
\text { - Customers resistance towards } \\
\text { ERP in the cloud } \\
\text { [24],[23],[22],[33] } \\
\text { - Organizational Change } \\
\text { [17],[4],[6] } \\
\text { - Increasing complexity [20],[4] }\end{array}$ & $\begin{array}{l}\text { Category D: External-Operational } \\
\text { challenge } \\
\text { - Performance [4],[23],[33],[25],,[28],[40] } \\
\text { - Functionality Fit [17],[4],[33],[23],,28] } \\
\text { - Monitoring, Analysis and Building Trust } \\
\text { [ } 39],[4],[25],[28] \\
\text { - Having a standard for ERP in the cloud } \\
\text { [24],,44],[28] } \\
\text { - Data Migration/extraction [17],[4],[25] } \\
\text { - Latency [21],,23] }\end{array}$ \\
\hline & & $\begin{array}{l}\frac{\text { Category E: Internal-Technical }}{\text { challenge }} \\
\text { - Network and internet } \\
\text { [2],[4],[45],,[23],[41],[22] } \\
\text { - Customization } \\
{[4],[22],[33],[23],[41],[3],[25],[2} \\
\text { 8],[40] } \\
\text { - Loss of IT competencies } \\
\text { [4],[23],[28] }\end{array}$ & $\begin{array}{l}\text { Category F: External-Technical challenge } \\
\text { - Security and privacy [17],[2],[20],[4],[44], } \\
\text { [22],[45],[23],[41],[3],[25],[28],[40] } \\
\text { - Elasticity and Scalability } \\
\text { [2],[39],[22],[23],[25] } \\
\text { - Integration [17],[4],[33],[23],[28] } \\
\text { - Reliability of systems [17],[23],[3],[4] } \\
\text { - Multiple Locations for Storage } \\
\text { [2],[22],[45],[44] } \\
\text { - Issues related to mobile access and } \\
\text { provisioning [39],[25] } \\
\text { - Technological Bottlenecks [39],,[25] } \\
\text { - Denial of Service [2] } \\
\text { - Upgrades of the system [33] }\end{array}$ \\
\hline
\end{tabular}

Figure 3. A Taxonomy Showing Six Categories of Cloud ERP Implementation Challenges

Out of thirty-one challenges, eighteen challenges are external (e.g. security and privacy, integration) in nature, whereas thirteen challenges are internal (e.g. resistance from end-users, organizational change) in nature. We now discuss the characteristics of each category:

Category A (Internal strategic challenges): Organizations encountering this category of challenges have strategic difficulties perceived by internal stakeholders. Strategic risk is a frequently mentioned challenge in this category (mentioned by two papers). client organizations heavily depend on Cloud ERP vendors since everything about Cloud ERP is handled by them [24]. Therefore, the client organizations' strategic risk increases when they are tied to a specific vendor [28]. Furthermore, other challenges cannot be ignored. For example, vendors play a significant role in implementing Cloud ERP since they have multiple responsibilities, such as providing hardware and software, monitoring the system, supporting users. As a result, proper selection of vendors with long-standing position in the market and strong background in Cloud ERP is always regarded as the matter of concern for client organizations [4]. As a result, selecting the vendor with high integrity is one of the internal strategic challenges.

Category B (External strategic challenges): Organizations encountering this category of challenges have external strategic difficulties perceived by external stakeholders. The most frequently reported challenge in this category is compliance risks, which has been mentioned by four papers. Cloud ERP vendors are required to provide their systems to comply with the rules, regulations and specifications laid down by the governments. This is because these rules can facilitate integrity and security of organizational information security. However, different countries have various types of laws and regulations. It is difficult for Cloud ERP vendors to adapt their systems to follow all country-based or location-based rules or regulations [4]. Furthermore, most organizations are still unwilling to apply Cloud ERP since they are not familiar with the impact of implementing this new technology. As a result, Cloud ERP vendors should put more focus on improving their business strategy on describing Cloud ERP services and making it clearer for their clients [28].

Category $C$ (Internal operational challenges): Organizations encountering this category of challenge have internal operational difficulties perceived by internal stakeholders. Challenges, like SLAs and longterm costs, are frequently mentioned in this category (please refer to Section 3.1 for clarification). For example, it is quite a challenge for client organizations to adopt Cloud ERP and abandon their existing onpremise system. This is because Cloud ERP not only requires employees to adjust their routines to new ways of working but also may result in job losses since a lot of work is outsourced to third-parties [28]. This can be a significant challenge for large organizations as they need to invest significant resources and time to train everybody [17]. As a result, organizational change is an internal operational challenge.

Category D (External operational challenges): Organizations encountering this category of challenges have external operational difficulties perceived by external stakeholders. Performance and functional fit are the most frequently mentioned challenges in this category. Inadequate Cloud ERP performance can have a negative influence on some important financial and operational decisions for client organizations [4]. However, client organizations cannot control the Cloud ERP systems because of their characteristics. It is a challenge for Cloud ERP vendors to deliver high quality product package to their clients [24]. Furthermore, complying with data, power and environmental standards are other difficulties faced by Cloud ERP vendors since the physical system may be located anywhere in the world [28]. It is difficult for Cloud ERP vendors to provide a unified system that adapts 
individual security policies to the specific country regulations [23].

Category E (Internal technical challenges): Organizations encountering this category of challenge have internal technical difficulties perceived by internal stakeholders. The most frequently reported challenges in this category is customization, network and internet (please refer to Section 3.1 to get more information). Furthermore, client organizations may lose some valuable IT competencies as they outsource a major part of their IT support [30]. When they depend heavily on Cloud ERP vendors, they may face challenges to be in sync with IT capabilities of vendors [4]. As a result, client organizations also need to consider how to overcome this challenge when they decide to outsource their IT support to third parties.

Category $F$ (External technical challenges): Organizations encountering this category of challenge have external technical difficulties perceived by external stakeholders. Security and privacy is found to be a considerably reported challenge in this category (please refer to Section 3.1 for more clarification). Another frequently reported challenge is elasticity and scalability. Elastic demands and services in the cloud computing environment increase the complexity of systems, which requires the involvement of more highly skilled technical people to develop and maintain systems [26]. Moreover, Mac-Anigboro and Usoro [24] suggest unscalable storage of systems is one of the disadvantages of implementing Cloud ERP systems because storage facilities are unable to be increased based on the requirements of client organizations. Accordingly, elasticity and scalability are considered as challenges of Cloud ERP vendors.

\section{Discussion}

\subsection{Cloud ERP implementation challenges}

From our systematic literature review on challenges of Cloud ERP implementation, the top five most widely cited challenges for Cloud ERP implementation are: security and privacy, customization, network and internet, SLA and long-term costs. Lesser, but still frequently mentioned challenges are performance, elasticity and scalability, functionality fit, integration and compliance. Comparing these findings with top 10 challenges for on-premise ERP implementation identified by, Mahmood and Khan [31] challenges for Cloud ERP and on-premise ERP differ and there exists only one commonality: integration is considered as a significant challenge for both of them. Although an ERP system (i.e. both on-premise and Cloud) is considered as a cross-functional enterprise system that integrates organizational core business processes, no single application can provide everything for organizations to fulfill their requirements [31]. Integration may happen at either in a single organization or across organization boundaries since organizations may purchase different modules from different vendors. If there is not a proper integration strategy, it can result in data loss, new system delays [32]. However, different challenges are identified due to the nature of Cloud ERP. For example, allowing customization is considered as one of advantages for on-premise ERP, but Cloud ERP does not provide space for extensive customization. This is because organizations have ownership of the software to customize systems as needed in on-premise ERP systems, whereas the ownership in Cloud ERP systems is controlled by vendors [1]. Therefore, on-premise ERP is more appropriate to some organizations with multiple customization requirements. Furthermore, compared to on-premise ERP, project management is no longer a big challenge for Cloud ERP implementation since client organizations do not need to initiate, plan, develop systems by themselves and the systems apply pay-peruse strategy [4]. As a result, Cloud ERP is always attractive to SMEs [5].

Only a few existing scholars offer solutions for some challenges of Cloud ERP implementation. For example, Ambavane and Pawar [20] recommend the adoption of security strategies and regular security assessment should be introduced to reduce the security issues occurred in implementing Cloud ERP. In another study, Rabaya and Graffi [25] suggest regular monitoring and measure from vendor and client sides is an effective way to make sure the quality of SLAs.

\subsection{Inconsistency issues about Cloud ERP implementation challenges}

It can be seen that challenges affecting Cloud ERP are inconsistent due to three reasons. Firstly, the inconsistent findings about the same challenge results from different perspectives. For example, updates have been considered as a benefit of implementing Cloud ERP by the cloud providers [28]. However, Iqbal and Uppström [35] regard upgrades as a challenge for client organizations. This is because the cloud providers are only responsible for handling the technical side whereas the users have to handle the functional side. Secondly, different Cloud models contribute to the differences in the challenges. For example, according to some researchers, Cloud ERP systems offer very limited customization options to client organizations as they are developed on SaaS layer of cloud computing which is assumed to be less flexible [4]. However, findings of the study conducted by Iqbal et al. (2012) suggest customization is not a big issue in Cloud ERP systems due to cloud providers offering platforms (i.e. PaaS, 
IaaS) that allow client organizations to build their codes and run them. Thirdly, the inconsistent findings about the same challenge results from different components consists of them. For example, IT cost reduction is considered to be the dominant benefit of Cloud ERP. This is because the cost of maintenance, configuration, and network etc. is reduced. Start-up and operating costs are also reduced [24, 28]. However, the cost of Cloud ERP is also considered as a challenge of implementing Cloud ERP. For example, some costs, such as transition cost, monitoring cost, and update cost are hidden in the contract, which will increase the fixed costs [28].

\subsection{The six categories of Cloud ERP implementation challenges}

Existing literature often applies the "Locus (source) of challenge" to comprehensively understand the implementation of complex systems that may involve more than one stakeholder, e.g. e-commerce [33]. Although the locus of challenges is not explicitly discussed in the existing Cloud ERP literature, it is an important characteristic to describe challenges in this area. The main Cloud ERP stakeholders are client organizations and Cloud ERP vendors. Existing literature suggests that common challenges include internal and external challenges in an organization and cannot be overlooked while implementing Cloud ERP [34]. It is then appropriate that the locus of challenges is identified as an important dimension for the taxonomy.

In the taxonomy, most of the strategic challenges are related to internal locus, thus decision makers in the client organizations are required to consider several strategic issues associated with the adoption and implementation decision of Cloud ERP. For example, they should select an appropriate vendor in terms of their financial background, integrity, reliability, prices, and user reviews [4]. They may also take some strategic risks (e.g. vendor lock-in) resulting from outsourcing their core IT technologies [3]. Some strategic challenges also are related to external locus, where vendors aim to attract more customers by adapting regulations or rules and improving their business strategy [4, 28]. Operational challenges are mentioned in both internal and external loci. As a result, successful implementation of Cloud ERP requires significant attention from both client organizations and Cloud ERP vendors through addressing these operational challenges [1]. For example, one of the operational challenges for client organizations is increasing complexity. Cloud ERP may need to expand to new geographies or to multiple departments, which will further increase the complexity of the system. It also can be difficult for Cloud ERP to adjust these changes made in real-time basis [4]. As a result, it is challenging for client organizations to reduce the complexity of Cloud ERP resulting from their business complexity. Since Cloud ERP is not mature enough, the existing functions are not extensive enough to cater for all of requirements in every type of industry [35]. Furthermore, some Cloud-based applications are only available to certain geographical locations because they cannot meet the financial reporting requirements of every region [36]. Accordingly, it is challenging for Cloud ERP vendors to enhance the capability of their systems. Most of the technical challenges are related to external locus, so the main responsibility of Cloud ERP vendors is to host the function and the quality of systems for all client organizations [3, 14]. For example, Cloud ERP allows users to access services everywhere through several devices, which requires Cloud ERP vendors integrate cutting edge mobile technologies with their Cloud ERP technologies [37].

Unlike Cloud ERP, the challenges identified from on-premise ERP implementation were categorized and fall within the client organizations, which includes operational, organizational and technical categories [38]. This is because on-premise ERP is developed by client organizations themselves [34]. Furthermore, highly related challenges of on-premise ERP implementation are related to the human aspect rather than technical issues [38]. However, given the complexity of Cloud ERP implementation, the highly related challenges of Cloud ERP implementation are both related to human and technical aspects.

\subsection{The common challenges for the stakeholders}

We further argue that different stakeholders are likely to experience some of the same Cloud ERP challenges. In other words, some challenges (e.g. strategic risk) can be addressed within the organization whereas some require cooperation between internal and external organizations. For example, both internal and external operational challenges can be encountered. Organizations encountering these two categories of challenge have operational difficulties perceived by both internal and external stakeholders. Service Level Agreements (SLAs) that are used to define the specifics of IT services delivered by providers to particular customers, which are considered as insufficient to cover the huge and hidden details of ERP systems implementation [25]. This is because the Cloud operates in an environment that spans geographies, networks and systems [3]. Challenges can result from many sources, including network, regulations, storage and power. It is a challenge for Cloud ERP vendors to propose SLAs to cover all details of the implementation issue whereas it is hard for client organizations to monitor and evaluate SLAs all the time [39]. Furthermore, given the vendor 
has access to the entire data of client organizations using its leased services, the management and monitoring of important assets (e.g. customer data, information about products and transactions) is not controlled by client organizations. This may lead to information theft or loss and result in financial loss [28]. As a result, it is essential for client organizations to have the requisite manpower to monitor the way the Cloud vendor deals with their data and also for Cloud ERP vendors to demonstrate their platform is secure and complies with the legal regulations proposed by the industry and the country [4].

Organizations encountering these two categories of challenges also have technical difficulties perceived by both internal and external stakeholders. Cloud ERP systems depend heavily on high-speed Internet quality as any network failures may disrupt the usual business function of client organizations [24, 40]. As a result, it is a mandatory requirement for both client organizations and vendors to maintain a high-speed network to ensure Cloud ERP implementation [2]. Furthermore, customization cannot be avoided for some client organizations because achieving alignment between the business process and Cloud ERP is required [22]. However, Cloud ERP with heavy customization would increase the cost of update and maintenance for clients [41]. On the other side, Cloud ERP vendors tend to keep customization at a minimal level to ensure standardization to meet the needs of more customers [3]. Vendors will accept customization if functional aspects of their products are similar to other existing ones in the market, which can improve their competitive advantage through high levels of customization competency [42]. As a result, it is necessary to both client organizations and vendors to have a negotiation on the level of customization.

\section{Conclusion}

This paper aims at developing a taxonomy for Cloud ERP challenges. Drawing on a systematic

\section{References}

[1] Peng, G.C.A. and C. Gala, Cloud ERP: a new dilemma to modern organisations? Journal of Computer Information Systems, 2014. 54(4): p. 22-30.

[2] Chand, S., et al. Cloud ERP Implementation Using Edge Computing. in 2018 5th Asia-Pacific World Congress on Computer Science and Engineering (APWC on CSE). 2018. IEEE.

[3] Vadivelu, K., et al., cloud-erp: implementation strategies, benefits and challenges. International Journal of Pure and Applied Mathematics, 2018. 119(14): p. 1359-1364.

[4] Gupta, S., et al., Identification of challenges and their ranking in the implementation of cloud ERP: A comparative study for SMEs and large organizations. analysis of the relevant literature, a set of seventeen (17) papers has been identified that focus on challenges arising from Cloud ERP implementation. Thirty-one challenges have so far been scrutinized through SLR and some interesting insights were provided. Firstly, the top five challenges were identified: security and privacy, customization, network and internet, SLAs and long-term costs. Secondly, the taxonomy has been developed with two dimensions. Most of the strategic challenges are faced by the internal stakeholder whereas operational and technical challenges require both internal and external stakeholders' attention. Some challenges are the same for both internal and external stakeholders (e.g., SLAs, network and the Internet). One future direction of research that we recommend is to further evaluate our assertion (outlined in Section 3.2) about the lack of influence of organization size on stakeholders' perceptions of challenges.

An important contribution of this research is a proposed taxonomy of Cloud ERP that is expressed in terms of two dimensions: type of challenges and locus of challenges. This taxonomy serves as a springboard for extending further research by developing higherlevel theories (e.g., Type II: a theory for explaining) that are linked to each of the six categories presented in our taxonomy (Figure 3). In practice, the findings are useful for main stakeholders involved in Cloud ERP implementation including client organizations and vendors to develop measures or strategies to overcome challenges in terms of the locus of challenges, which can enhance the possibility of successful Cloud ERP implementation. For example, IT staff in the vendor side should pay more attention to the external technical challenges, especially security and privacy, customization issues since they are more frequently mentioned. This study developed the taxonomy of cloud ERP implementation challenges based on the existing literature; thus, future research should assess this taxonomy by collecting empirical data.

International Journal of Quality \& Reliability Management, 2017. 34(7): p. 1056-1072.

[5] Hentschel, R., C. Leyh, and T. Baumhauer. Critical success factors for the implementation and adoption of cloud services in SMEs. in Proceedings of the 52nd Hawaii International Conference on System Sciences. 2019.

[6] Lim, W.M. and D.H. Ting, Consumer acceptance and continuance of online group buying. Journal of Computer Information Systems, 2014. 54(3): p. 87-96.

[7] Fleck, J., Learning by trying: the implementation of configurational technology. Research policy, 1994. 23(6): p. 637-652.

[8] Huang, Q., et al. Critical Success Factors Affecting Implementation of Cloud ERP Systems: A Systematic Literature Review with Future Research Possibilities. 
in Proceedings of the 54th Hawaii International Conference on System Sciences. 2021.

[9] Ahmed, R., G. Oliver, and M. Rahim, Understanding Potentials of Cloud ERP Adoption by Large Organisations: A Case Study. 2020.

[10] Huang, Q., et al., understanding cloud-based erp customization from key stakeholders'perspectives: a research model. 2021.

[11] Bender, B., C. Bertheau, and N. Gronau. Future ERP Systems: A research agenda. in Procs of $23 \mathrm{rd}$ International Conference on Enterprise Information Systems. 2021.

[12] Drasch, B.J., A. Schweizer, and N. Urbach, Integrating the 'Troublemakers': A taxonomy for cooperation between banks and fintechs. Journal of Economics and Business, 2018. 100: p. 26-42.

[13] Gregor, S., The nature of theory in information systems. MIS quarterly, 2006: p. 611-642.

[14] Song, H., et al. How to support customisation on SaaS: a grounded theory from customisation consultants. in 2017 IEEE/ACM 39th International Conference on Software Engineering Companion (ICSE-C). 2017. IEEE.

[15] Moucheraud, C., et al., Sustainability of health information systems: a three-country qualitative study in southern Africa. BMC health services research, 2017. 17(1): p. 23.

[16] Jones, O. and C. Gatrell, The future of writing and reviewing for IJMR. International Journal of Management Reviews, 2014. 16(3): p. 249-264.

[17] Sørheller, V.U., et al., Implementing cloud ERP solutions: a review of sociotechnical concerns. Procedia computer science, 2018. 138: p. 470-477.

[18] Jöhnk, J., et al., How to implement agile IT setups: a taxonomy of design options. 2017.

[19] Bailey, K.D., Typologies and taxonomies: An introduction to classification techniques. Vol. 102. 1994: Sage.

[20] Nickerson, R.C., U. Varshney, and J. Muntermann, $A$ method for taxonomy development and its application in information systems. European Journal of Information Systems, 2013. 22(3): p. 336-359.

[21] Ambavane, S.A., et al., Cloud Computing and SaaS (ERP) Implementation. 2018.

[22] Nowak, D. and K. Kurbel. Understanding the flexibility of cloud ERP software. in International Conference on Enterprise Resource Planning Systems. 2016. Springer.

[23] Appandairajan, P., N.Z.A. Khan, and M. Madiajagan. ERP on Cloud: Implementation strategies and challenges. in 2012 International Conference on Cloud Computing Technologies, Applications and Management (ICCCTAM). 2012. IEEE.

[24] Mac-Anigboro, O. and A. Usoro, Usability in Ensuring a Successful Post Implementation Adoption of Cloud Enterprise Resource Planning Systems: A Proposed Research. Computing \& Information Systems, 2015. 19(2).

[25] Rabaya, A. and K. Graffi. Implementing Enterprise Resource Planning Systems in the Cloud: Challenges and Solutions. in 2019 International Symposium on
Networks, Computers and Communications (ISNCC). 2019. IEEE

[26] Purohit, G., M. Jaiswal, and S. Pandey, Challenges involved in implementation of ERP on demand solution: Cloud computing. International Journal of Computer Science Issues (IJCSI), 2012. 9(4): p. 481.

[27] Haas, P., I. Blohm, and J.M. Leimeister, An empirical taxonomy of crowdfunding intermediaries. 2014.

[28] Abd Elmonem, M.A., E.S. Nasr, and M.H. Geith, Benefits and challenges of cloud ERP systems-a systematic literature review. Future Computing and Informatics Journal, 2016. 1(1-2): p. 1-9.

[29] Hustad, E., et al. Creating Business Value from CloudBased ERP Systems in Small and Medium-Sized Enterprises. in Conference on e-Business, e-Services and $e$-Society. 2019. Springer.

[30] Jlelaty, M. and Y. Monzer, Factors in Cloud Computing Adoption, Lund University, School of Economics and Management. Information Systems Department, 2012.

[31] Ali, M. and L. Miller, ERP system implementation in large enterprises-a systematic literature review. Journal of Enterprise Information Management, 2017.

[32] Barki, H. and A. Pinsonneault, A model of organizational integration, implementation effort, and performance. Organization science, 2005. 16(2): p. 165-179.

[33] Quaddus, M. and D. Achjari, A model for electronic commerce success. Telecommunications Policy, 2005. 29(2-3): p. 127-152.

[34] Khaparde, V.M., Barriers of ERP while implementing ERP: a Literature Review. Journal of Mechanical and Civil Engineering, 2012. 3(6): p. 49-91.

[35] Scavo, F., B. Newton, and M. Longwell, Choosing between cloud and hosted ERP, and why it matters. Computer Economics Report, 2012. 34(8): p. 1.

[36] Utzig, C., et al., ERP in the cloud is it ready? Are you? Perspective, 2013: p. 1-9.

[37] Goel, M.S., R. Kiran, and D. Garg, Impact of cloud computing on ERP implementations in higher education. Institutions, 2011. 5: p. 8.

[38] Mahmood, F., A.Z. Khan, and R.H. Bokhari, ERP issues and challenges: a research synthesis. Kybernetes, 2019.

[39] Bhadra, S., M.K. Sanyal, and B. Biswas. Cloud ERP Adoption Pitfalls and Challenges-A Fishikawa Analysis in the Context of the Global Enterprises. in International Conference on Computational Intelligence, Communications, and Business Analytics. 2018. Springer.

[40] Navaneethakrishnan, C., A comparative study of cloud based ERP systems with traditional ERP and analysis of cloud ERP implementation. International Journal of Engineering and Computer Science, 2013. 2(9): p. 2866-2869.

[41] Uppström, E., et al. New implications for customization of ERP systems. in 2015 48th Hawaii International Conference on System Sciences. 2015. IEEE.

[42] Sun, W., et al. Software as a service: Configuration and customization perspectives. in 2008 ieee congress on services part ii (services-2 2008). 2008. IEEE. 